\title{
Study of the twinned dendrite tip shape II: Experimental assessment
}

\author{
M.A. Salgado-Ordorica ${ }^{\mathrm{a}, *}$, P. Burdet $^{\mathrm{b}}$, M. Cantoni ${ }^{\mathrm{b}}$, M. Rappaz $^{\mathrm{a}}$ \\ ${ }^{a}$ Laboratoire de Simulation des Matériaux LSMX, Ecole Polytechnique Fédérale de Lausanne, Station 12, 1015 Lausanne, Switzerland \\ ${ }^{\mathrm{b}}$ Centre Interdisciplinaire de Microscopie Electronique CIME, Ecole Polytechnique Fédérale de Lausanne, Station 12, 1015 Lausanne, Switzerland
}

\begin{abstract}
The favorable growth kinetics of twinned dendrites can be explained by their complex morphology, multiple side branching mechanisms, growth undercooling and tip morphology. Three models were proposed for the twinned dendrite tip shape: (i) a grooved tip [1] satisfying the Smith condition at the triple line; (ii) a doublon [2], i.e. a double-tip dendrite that grows with a narrow and deep liquid channel in its center; and (iii) a pointed (or edgy) tip [3], with consideration of the solid-liquid interfacial energy anisotropy. In the first part of this work, phase field simulations of half a twinned dendrite with an appropriate boundary condition to reproduce the Smith condition supported the doublon conjecture, with a narrow liquid channel ending its solidification with the formation of small liquid droplets. In this part, experimental observations of twinned dendrite tips reveal the presence of a small, but well-defined, groove, thus definitely eliminating the edged tip hypothesis. Focused ion beam nanotomography and energy-dispersive spectroscopy chemical analysis in a transmission electron microscope reveal the existence of a positive solute gradient in a region localized within $2 \mu \mathrm{m}$ around the twin plane. In $\mathrm{Al}-\mathrm{Zn}$ specimens, small particles aligned within the twin plane further support the doublon conjecture and the predicted formation of small liquid droplets below the doublon root.
\end{abstract}

(C) 2011 Acta Materialia Inc. Published by Elsevier Ltd. All rights reserved.

Keywords: Twinned dendrites; Doublon growth; Focused ion beam; Aluminum alloys

\section{Introduction}

As presented in the companion paper [4], three conjectures have been put forward for the tip shape of $\langle 110\rangle$ twinned dendrites in order to explain their growth advantage over regular $\langle 100\rangle$ dendrites in $\mathrm{Al}$ alloys: (i) a dendrite tip with a groove that is required to satisfy the Smith equation at the triple line between the twinned solid, the untwinned solid and the liquid [1]; (ii) a dendrite tip split in its center by a thin liquid pool, also called a doublon, due to solute pile-up ahead of a grooved tip [2]; and (iii) an edgy tip with consideration of torque terms induced by the anisotropy of the solid-liquid interfacial energy $\gamma_{s \ell}$ [3]. An edgy tip would definitely give a growth advantage to twinned dendrites over regular ones, but this shape is incompatible with the very weak anisotropy measured

\footnotetext{
* Corresponding author. Present address: Novelis Switzerland SA, Route de Laminoires 15, CH-3960 Sierre, Switzerland.

E-mail address: mario.salgado@novelis.com (M.A. Salgado-Ordorica).
}

recently for $\gamma_{s \ell}$ in $\mathrm{Al}-\mathrm{Cu}$ alloys [5]. On the other hand, a steady-state grooved dendrite tip would make solute diffusion more difficult and thus should not give it a growth advantage. Finally, the doublon-type dendrite tip is compatible with the Smith equation at the root of the doublon, while drastically changing solute rejection near the tip.

Based on several metallographic observations and segregation analyses performed on twinned dendrites of directionally solidified (DS) industrial alloys, Henry [2] gave three experimental arguments in favor of the doublon morphology: (i) the primary dendrite trunk spacing measured along the twin plane $\left(\lambda_{/ /}\right)$is much smaller than that perpendicular to it $\left(\lambda_{\perp}\right)$. Doublons at the center of dendrite trunks, all aligned along a (111) twin plane, would definitely affect solute diffusion parallel and perpendicular to the twin plane, and thus the primary trunk spacing; (ii) besides the twin relationship between successive lamellae, the dendritic structure in these lamellae exhibits a gradual and systematic misorientation which is responsible for the fan-shape appearance of feathery grains $[2,6-8]$. A strong 
solute gradient perpendicular to the twin planes and associated with doublons at the center of twinned dendrites can induce a gradual rotation of successive twin planes during branching when the solute element has a radius different from that of the solvent; and (iii) using highresolution transmission electron microscopy (HRTEM) in a region near to a coherent twin plane, Henry measured a composition of the solid close to the nominal composition $C_{0}$ of the alloy, instead of a value close to $k_{0} C_{0}$, where $k_{0}$ is the partition coefficient [2]. However, these measurements were made in industrial alloys of fairly low composition ( $\mathrm{Al}-4.3$ wt. $\% \mathrm{Cu}-0.3 \mathrm{wt} . \% \mathrm{mg}$ ).

Under a high thermal gradient, the growth conditions can be close to those of the cellular regime. This might explain why Henry observed in these alloys cells in sections nearly parallel to the twin plane and dendrites perpendicular to it, with the consequence that $\lambda_{/ /} \ll \lambda_{\perp}$. In previous works, Salgado-Ordorica and Rappaz [6,7] showed that, in binary $\mathrm{Al}$ alloys with relatively high solute-content, $\lambda_{/ /}$ is comparable to $\lambda_{\perp}$, even though dendrites trunks were aligned along the twin planes. The first argument of Henry is thus put into question. The third question is also unclear, since the present authors [6] did not find any increase in segregation near the twin plane in $\mathrm{Al}-30 \mathrm{wt} . \% \mathrm{Zn}$ twinned dendrites. However, this was done on a transverse section of an specimen using wavelength-dispersive X-ray spectroscopy (WDS) chemical analysis in a scanning electron microscope, and not by HRTEM, and thus the resolution was possibly insufficient. Furthermore, the specimens were not quenched, and back-diffusion could have smeared any sharp segregation profile during cooling.

Using a phase field model, the first part of this work clearly shows that the small groove necessary to satisfy the equilibrium condition at the triple line rapidly evolves into a doublon morphology. It also predicts that the width of the narrow liquid channel of a doublon might be a few microns only, and that solute diffusion during and especially after solidification indeed smears out the solute gradient in the region close to the twin plane. Here, further experimental evidence is presented that supports, if not definitely proves, the doublon morphology of twinned dendrite tips. These experiments include: (i) observations of the twinned dendrite tip shape in regions where its growth was abruptly stopped by impingement with another grain; and (ii) three-dimensional (3-D) reconstruction, using focused ion beam (FIB) nanotomography, of a small volume element containing a dendrite trunk center, with simultaneous scanning transmission electron microscopy (STEM) and energy-dispersive spectroscopy (EDS) analyses.

\section{Experiments and methods}

The $\mathrm{Al}-\mathrm{Cu}$ and $\mathrm{Al}-\mathrm{Zn}$ binary alloys used in this study were produced from a mixture of their pure elements ( $99.997 \mathrm{wt} . \%$ purity) in the directional solidification installation, with and without a quenching device, as described in [6] and [7]. After appropriate chemical etching of selected regions of the solidified specimens, twinned dendrites were identified and studied using electron microscopy and electron backscatter diffraction measurements performed in a Philips FEI XLF30-FEG scanning electron microscope equipped with the appropriate detectors.

A Zeiss NVision 40 (CrossBeam) scanning electron microscope with a FIB (Ga liquid metal ion source) was used to perform serial sectioning of a volume of about $10 \times 10 \times 20 \mu^{3}$, taken from transverse sections of twinned dendrite trunks containing a coherent twin plane. More specifically, successive slices a few nanometers thick and $10 \times 20 \mu \mathrm{m}^{2}$ wide were removed by Ga ion bombardment, while scanning electron microscopy (SEM) images of the 2-D surface were recorded before each pass. The reconstruction was then made directly from the stack of 2-D images. Fig. 1 shows an SEM micrograph obtained before starting the serial "sectioning" procedure for an Al-6 wt.\% $\mathrm{Cu}$ specimen. The twin plane is indicated with a straight white line, while the thermal gradient $G$ direction when the specimen was directionally solidified is shown with an arrow.

A FIB was also used to extract a thin lamella for TEM analysis. The reason why the specimen shown in Fig. 1 was milled around the region of interest prior to serial "sectioning" was remove completely to facilitate the lamella extraction. The crystallographic structure of the specimen near the twin plane was first investigated by electron diffraction in a transmission electron microscope. Then image acquisition in STEM mode with an annular dark field (ADF) detector and EDS (Oxford Instruments INCA system) chemical analysis of regions near the twin plane were performed in a Philips/FEI CM300 FEG- $\mu$ T high-resolution scanning transmission electron microscope. As the necessary thickness of the sample for STEM analysis presents a number of complications when prepared by traditional methods (mechanical milling, chemical or ion etching), the FIB was a real asset to precisely locate the region of interest and produce samples of uniform thickness.

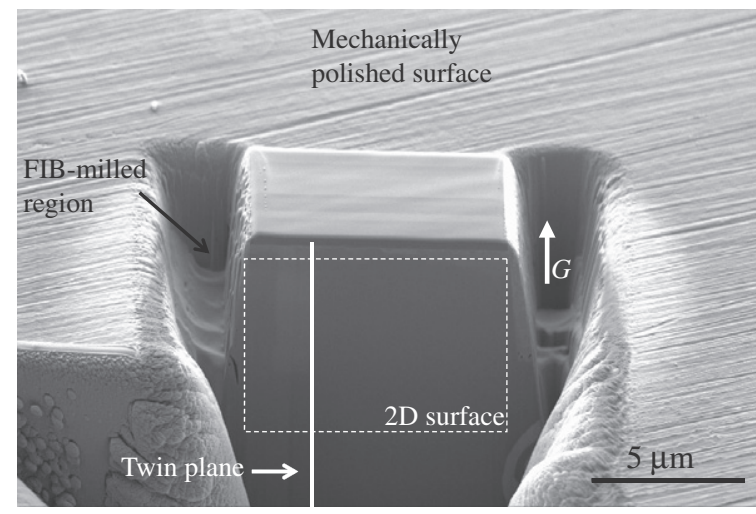

Fig. 1. Serial sectioning of an Al-6wt.\% $\mathrm{Cu}$ sample using a FIB. A dendrite trunk split by a coherent twin plane, indicated with a straight white line, is contained within the volume of interest. The transverse surface was mechanically polished, as evidenced by the scratches at the surface perpendicular to $G$. 


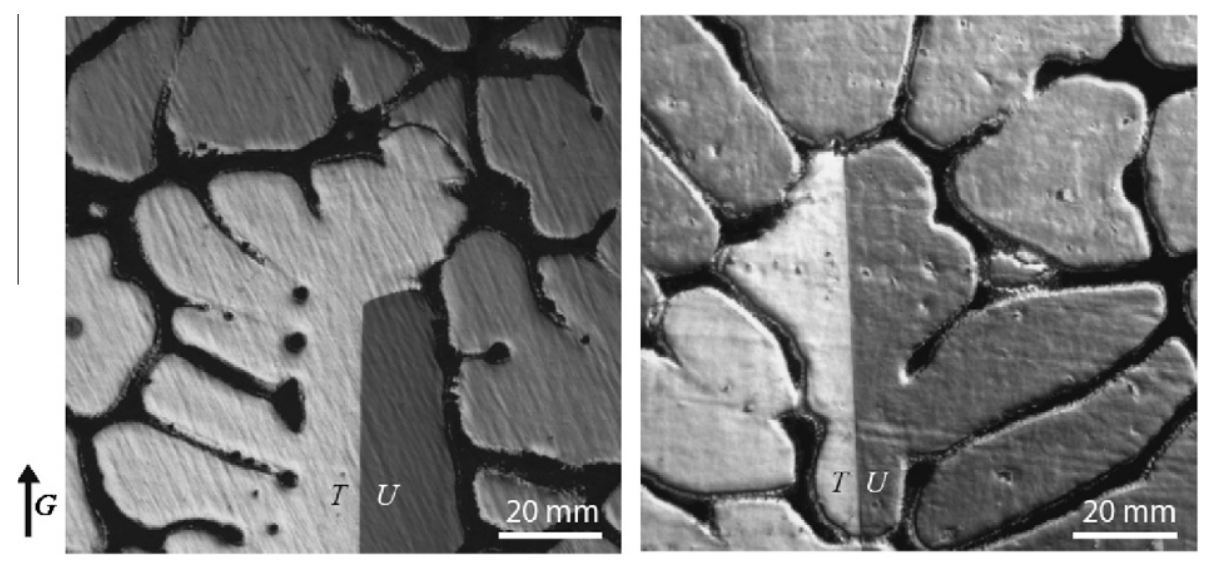

Fig. 2. Optical micrographs (Barker's etching) showing two twinned dendrite tips obtained in an Al-26 wt.\% Zn DS quenched specimen.

(a)
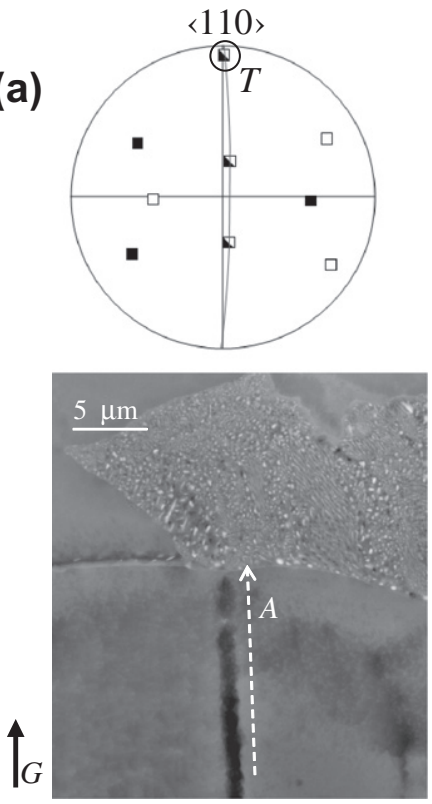

(b)
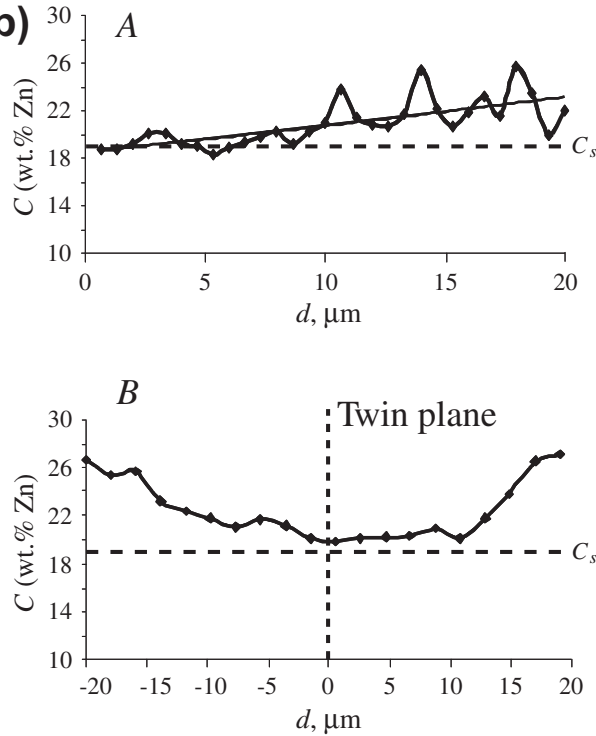

Fig. 3. (a) SEM-BSE micrograph of a twinned dendrite tip of an Al-30 wt. $\% \mathrm{Zn}$ specimen close to the region where it was stopped by an equiaxed grain. The thermal gradient $G$ is vertical. The $\langle 110\rangle$ pole figure indicates the orientation of the twin plane and the growth direction of the twinned dendrite trunk. The arrow $A$ shows the line along which the corresponding composition profile in (b) was measured using EDS. The composition profile (B) also shown in (b) was measured perpendicular to the twin plane.

\section{Results and discussion}

\subsection{Observations of "self-quenched" twinned dendrite tips}

Several attempts were made to visualize the shape of twinned dendrite tips in regular and quenched DS specimens. These observations were performed in regions of the specimen where a twinned dendrite impinged on either a regular dendrite or another twinned dendrite. They can be summarized by the two microstructures shown in Fig. 2. These were obtained for a DS Al-26 wt.\% Zn specimen quenched using the set-up described in Ref. [7]. The twinned $(\mathrm{T})$ and untwinned $(\mathrm{U})$ regions of a single twinned dendrite are clearly visible in these micrographs, the straight coherent twin plane being nearly perpendicular to the section. In the twinned dendrite shown on the left, the twinned part of the dendrite overgrows its untwinned part before it impinges on the equiaxed grain above. ${ }^{1}$ At the overgrowth location, the twinned-untwinned boundary makes an angle clearly smaller than $90^{\circ}$ with the vertical thermal gradient. This experimental evidence is not compatible with an edgy dendrite tip morphology. On the right of Fig. 2, the twinned and untwinned parts of the dendrite simultaneously impinge on the equiaxed grain formed above. A small groove is clearly apparent at the end of the twin plane, again in conflict with the edgy tip hypothesis.

\footnotetext{
${ }^{1}$ For further details on the overgrowth mechanisms please refer to [7] and [11].
} 
However, such observations do not allow any distinction between a grooved or doublon-type dendrite tip as a quench (even a fast one) cannot reveal a thin liquid channel 1 or $2 \mu \mathrm{m}$ wide, as predicted in the first part of this work.

An interesting measurement was also performed at the arrest point of a twinned dendrite by an equiaxed grain in an Al-30 wt.\% Zn specimen, as shown in Fig. 3a. This specimen was solidified in the DS installation without quenching. Since the micrograph was obtained with an SEM-backscattered electron (BSE) detector, the gray contrast is directly related to the composition, i.e. dark gray regions correspond to the primary aluminum phase and light regions to the $\mathrm{Zn}$-rich secondary phase. The twin plane appears as a thick black straight boundary and the $\langle 110\rangle$ pole figure on top of Fig. 3a shows its orientation. As can be seen, the $\langle 110\rangle$ trunk direction is parallel to the micrograph section (circle labeled " $T$ " in the pole figure). The precipitates region ahead of the twinned dendrite tip is made of a matrix of primary phase and small $\mathrm{Zn}$-rich light elongated particles. Chemical EDS measurements were performed in this section in directions parallel $(A)$ and perpendicular $(B)$ to the twin plane (see Fig. 3b). It should be noted that the composition profile $A$ was measured at about $2 \mu \mathrm{m}$ on the right of the twin plane, whereas the composition profile $B$ was measured in the specimen at about $50 \mu \mathrm{m}$ below the bottom of the micrograph. This last profile shows no sign of positive segregation near the dendrite trunk center, in agreement with our previous observations [6]. Indeed, considering a diffusion coefficient in the solid of about $3 \times 10^{-12} \mathrm{~m}^{2} \mathrm{~s}^{-1}$ and a doublon inner pool a few microns wide (see [10]), back-diffusion at high temperature occurs only within a few seconds and can therefore smear out any slight positive segregation within this region. On the other hand, the composition profile $(A)$ measured along the twin plane reveals a slight solute increase as one moves closer to the tip. However, this result is affected by the size of the interaction volume inherent to SEM measurements and thus the near $\mathrm{Zn}$-rich precipitates zone at the top. Additionally, in this impingement zone, the solute-content of the liquid must increase, as indicated by the precipitates zone, and so should the content of the last solidifying primary phase. More interesting are the composition oscillations shown by this profile. The peaks of this profile are higher than the solid composition $C_{s}$ calculated with the regular dendrite tip model of Kurz et al. [9] (the so-called KGT model for columnar dendrite growth) and indicated with a dashed horizontal line. These oscillations intensify as the end of the twin plane is reached and their spacing (about $\approx 3 \mu \mathrm{m}$ ) can be correlated with the periodic formation of small liquid droplets at the root of the doublon predicted by the phase field model in the first part of this study [10].

\subsection{FIB nanotomography}

An SEM micrograph of a transverse section from a twinned dendrite trunk of an Al-30 wt.\% Zn DS specimen is shown in Fig. 4a. The traces of $\langle 110\rangle$ arms in this section appear as dark uniform gray zones, with two-phase interdendritic zones in between apearing near each corner of

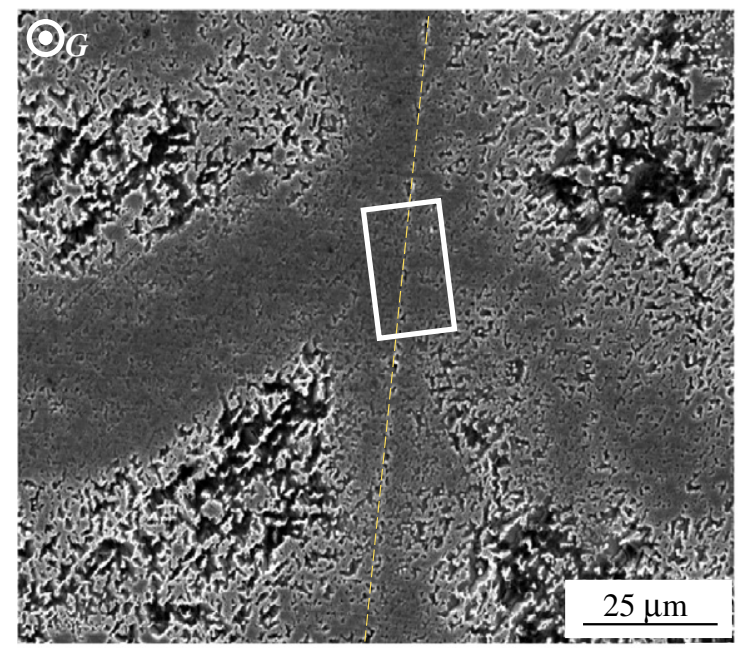

(a)

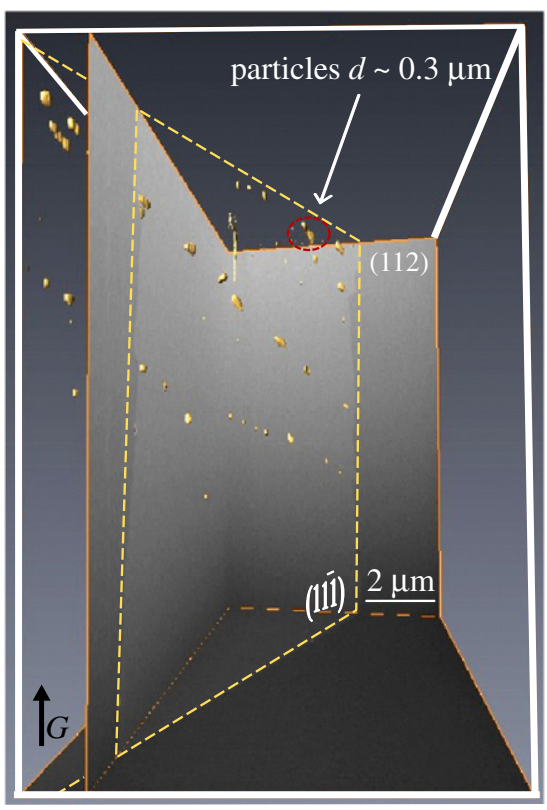

(b)

Fig. 4. (a) Transverse section of an Al-30 wt.\% Zn DS specimen. The small white rectangle indicates the region where a 3-D volume was reconstructed using FIB nanotomography. (b) Reconstructed volume showing the trace of the twin plane with yellow dashed lines. The slight gray contrast in the (112) plane identifies the twinned and untwinned lamellae of the twinned dendrite. The small particles appearing in yellow have a diameter of about $0.3 \mu \mathrm{m}$ and are distributed along the twin plane only. 
the figure. The small white rectangle in the center of the twinned dendrite trunk indicates the region containing the volume of $5 \times 10 \times 20 \mu^{3}$ reconstructed by FIB nanotomography in Fig. 4b. In this latter, the primary phase is presented as a transparent media, except for the (112) plane shown on the back. The different crystallographic orientations of this twinned dendrite can be distinguished from the slight gray contrast observed in this plane. The trace of the twin plane in the volume is indicated with yellow dashed lines, while a plane parallel to the left side of the volume helps visualize its extension. The observation of the various 2-D sections of the volume revealed the presence of small light regions that, due to the contrast, must be zinc-rich. In the reconstructed volume, these light regions can be seen as small yellow particles with a diameter of the order of $0.3 \mu \mathrm{m}$. More importantly, all these particles are aligned perfectly within the twin plane and do not appear anywhere else in the reconstructed volume.

It could be argued that these particles can form after solidification through a solid-state transformation, the twin boundary being a suitable nucleation site for any stable or metastable transformation occurring during cooling or even at room temperature. ${ }^{2}$ In recent work, Straumal et al. $[12,13]$ have shown that there is, indeed, a metastable transformation in the $\mathrm{Al}-\mathrm{Zn}$ phase diagram that can occur at a grain boundary a few degrees below the bulk solidus temperature $T_{s o l}$. Based on the concept of grain boundary phase transformations, introduced first by Cahn [14], these authors observed in Al-11.3 wt.\% Zn polycrystals annealed at a temperature a few degrees below $T_{\text {sol }}$ the formation of a hexagonal close-packed metastable phase at the grain boundary that contains about $45 \mathrm{wt} . \% \mathrm{Zn}$. This phase normally disappears during cooling, but in the specimen shown in Fig. 4 it could be stabilized at the twin boundary.

Fig. 5a shows another interesting feature of this reconstructed volume that might contradict this interpretation. It shows a vertical section perpendicular to the twin plane, i.e. corresponding to a (112) plane. The trace of the twin plane is visible by the difference in gray level between the two sides of the twinned dendrite. It is not straight, i.e. it is not fully coherent, but instead periodically exhibits ledges or stairs, i.e. it is only partially coherent. Furthermore, at some of the stairs, zinc-rich particles corresponding to the yellow particles evidenced in Fig. $4 \mathrm{~b}$ are present (one is circled in black in Fig. 5a). Although this ledge-like pattern is as yet unexplained, it seems to be associated with a local solute increase which, at some point, induces the precipitation of zinc-rich particles. This rather supports the conjecture that these particles actually formed during solidification, as they can be correlated with three phenomena: (i) the macroscopic progressive misorientation of

\footnotetext{
${ }^{2}$ The regular sequence of metastable phase formation at room temperature in the Al-Zn system is: Guinier-Preston (GP)-I zones (spherical preprecipitates coherent with the $\mathrm{Al}$ matrix), (GP)-II zones (semi-coherent ellipsoidal pre-precipitates), rhombohedral distorted face-centered cubic (fcc), distorted fcc and finally $\beta_{Z n}$ phase [12].
}

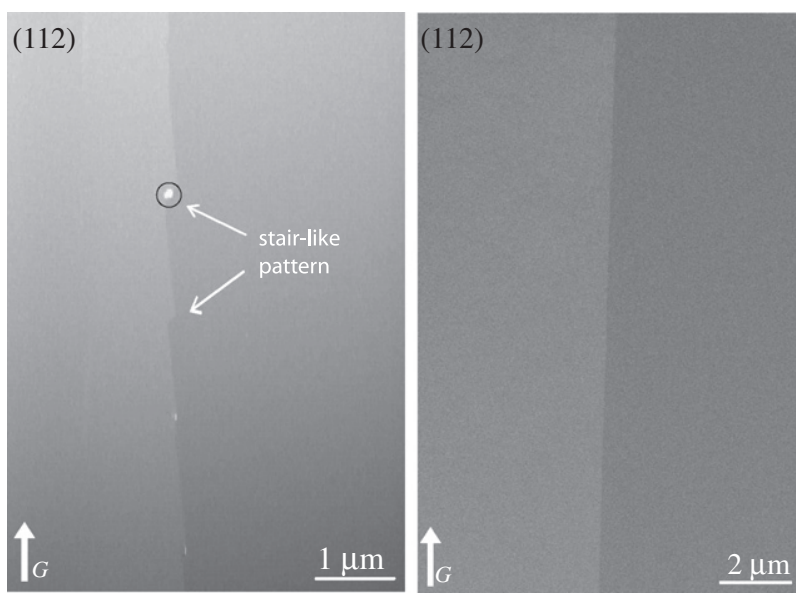

Fig. 5. (a) Ledge-type pattern along the twin plane of the Al-30 wt. $\% \mathrm{Zn}$ specimen shown in Fig. 4 in one of the sections obtained by FIB nanotomography perpendicular to the twin plane. The twinned and untwinned parts appear again with a slight gray contrast, while a small zinc-rich intermetallic particle appearing at one of the ledges (light region) is surrounded by a circle; (b) similar cross-section of a FIB reconstructed volume element for an Al- $6 \mathrm{wt} . \% \mathrm{Cu}$ specimen, in this case showing a fully coherent twin plane, with no particle along the twin plane.

lamellae within a twinned grain; (ii) the growth of faceted crystals which use ledges, among other mechanisms, to propagate a crystal along well-defined facets [15]; and (iii) the similarities it shares with the formation of lamellae during solid-state transformations in TiAl [16,17]. The $\alpha$-phase (hexagonal structure like $\mathrm{Zn}$ ) of TiAl can partially transform into $\gamma$-lamellae (fcc structure like $\mathrm{Al}$ ) by emitting new ledges at grain boundaries, which then grow within the $\alpha$-grains with a small solute pile-up ahead of it.

The same kind of FIB volume reconstruction and observation were performed for an Al-6 wt.\% $\mathrm{Cu}$ twinned specimen. One section of the reconstructed volume perpendicular to the twin plane is shown in Fig. 5b. In this case, we did not observe the presence of particles or ledges along the twin plane, i.e. the twin plane seems to be fully coherent in this alloy.

\subsection{STEM analysis}

A thin lamella was extracted using FIB from a transverse section of an Al-30 wt.\% $\mathrm{Zn}$ specimen. An SEM micrograph showing the zone of extraction is shown in Fig. 6a. The twin plane, clearly visible from the gray contrast, crosses the small extracted lamella at the center. Fig. $6 \mathrm{~b}$ shows an SEM micrograph of the thin lamella prior to EDS analysis in the STEM equipment, the contrast between the twinned and untwinned parts being barely visible. Due to its thickness, no Zn-rich particle was present in this lamella. A high-magnification image of the twin plane in the region shown with a square in this figure is presented in Fig. 6c (ADF detector). Note the small light regions only a few nanometers in length distributed along the twin plane. These are much smaller than the particles observed in the reconstructed volume of Fig. 4. Five composition 


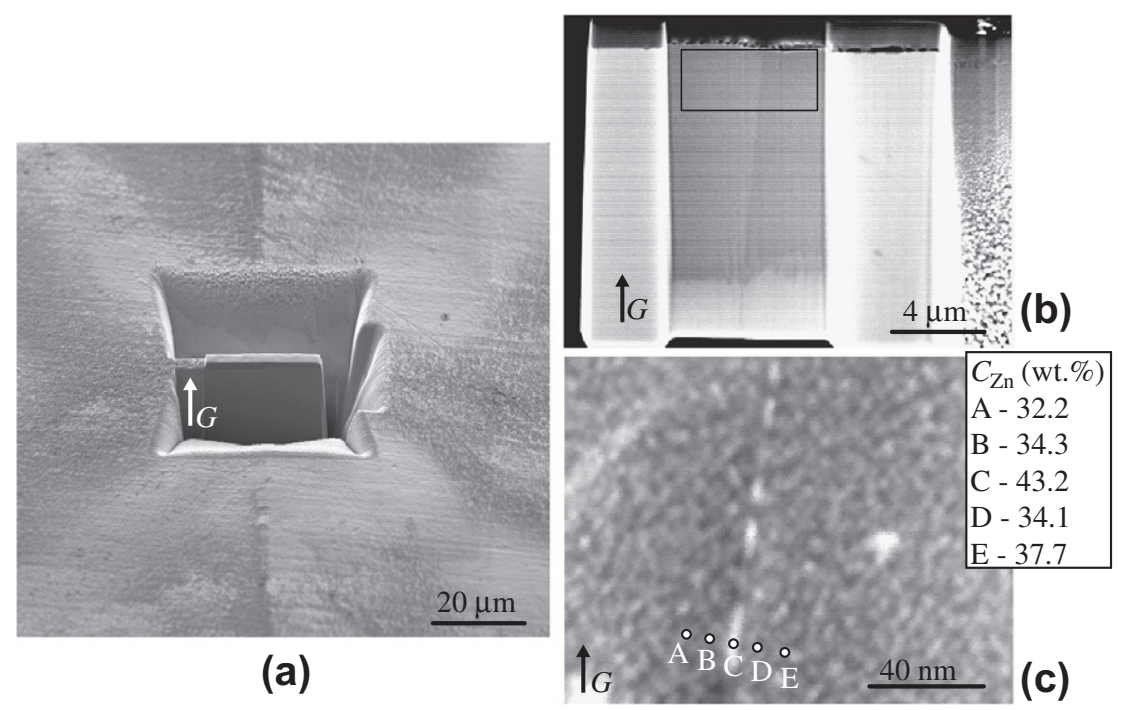

Fig. 6. (a) SEM micrograph showing the extraction region of a thin slice of an Al-30wt.\% Zn specimen for STEM and EDS analysis; (b) SEM micrograph of the thin slice after extraction. The gray contrast helps to identify the twin plane; (c) dark field TEM micrograph (ADF detector) of a region containing the twin plane.

(a)

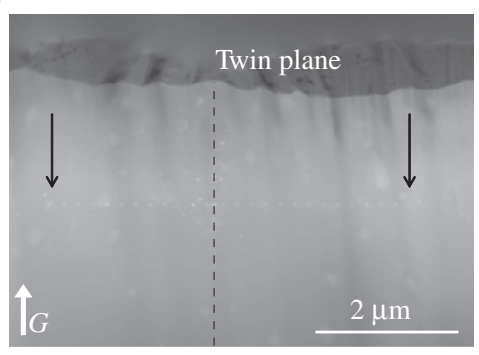

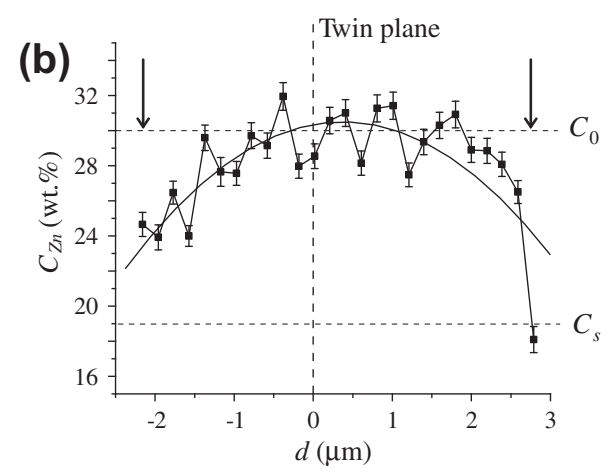

Fig. 7. (a) ADF micrograph of a thin slice of the Al-30 wt.\% Zn specimen shown in Fig. 6 . The black arrows indicate the starting and ending points (from left to right) of the sequence of small white spots where composition measurements were performed. The same arrows are shown in (b) on the actual composition profile measured in section (a). The smooth black curve is a second-order polynomial fit.

measurements were performed, and these are identified in the micrograph by the small white circles labeled A-E. The values are displayed in the inset table. The particle appearing precisely along the twin plane has a composition $C_{Z n}=43 \mathrm{wt} . \%$ and the neighboring spots show a composition above $32 \mathrm{wt} . \% \mathrm{Zn}$, which is higher than the nominal composition of the alloy. The composition higher than $C_{0}$ measured in this small region is probably due to solidstate transformations leading to the formation of a stable (or metastable) phase along the twin plane.

Since the STEM composition measurements are too localized near the twin plane, a second chemical analysis was performed at a much larger scale in the same thin slice. In the micrograph of Fig. 7a, two vertical arrows on either side of the twin plane (indicated with a dashed line) mark the start and end of a sequence of small white spots that correspond to the composition profile plotted in Fig. $7 \mathrm{~b}$. These measurements show some random fluctuations, while the continuous curve in the graph is a second-order polynomial least-squares fit. It can be observed that the composition in this region is higher than $C_{s}=19 \mathrm{wt} . \%$ $\mathrm{Zn}$, the solid composition calculated with the KGT model for the $\mathrm{Al}-30 \mathrm{wt} . \% \mathrm{Zn}$ alloy, and reaches a maximum close to $C_{0}$ near the twin plane. Moreover, an important negative solute gradient is measured on either side of the twin plane. This segregation profile certainly contradicts the results previously reported by the present authors in Ref. [6] and shown in Fig. 3b. In fact, the positive segregation evidenced in Fig. 7 occurs over a very narrow region near the twin plane, and is thus very difficult to elucidate through the SEM/EDS analysis presented in Fig. 3. In contrast, the regular spacing from the solute-rich particles observed along the twin plane in Figs. 5 and 6 can be correlated qualitatively with the composition oscillations observed in Fig. $3 \mathrm{~b}$ along the twin plane in a direction parallel to $G$. The STEM composition analysis confirms the TEM measurements of Henry [2] in low-solute-content Al alloys and support the doublon conjecture, i.e. the formation of a liquid channel of increasing composition at the center of the dendrite trunk. This is also consistent with 
the growth morphology predicted by the simulations. Indeed, the positive segregation measured in the twin plane can be related to the solute gradient observed within the solid phase below the doublon root in the simulations (see Fig. 9 of [10]), and could also account for the eventual formation of small particles in the Al- Zn alloy.

\section{Conclusions}

In the first part of this work [10], phase field simulations showed that a grooved dendrite tip is not stable and quickly degenerates into a doublon-type dendrite. In this part, several pieces of experimental evidence are presented which support these simulation results. First, direct observations of the shape of the primary phase morphology near a dendrite tip that was stopped by impingement on another grain reveal the presence of a small groove. This clearly eliminates the conjecture of an edgy tip suggested by Watson and Hunt [18], conjecture that is also incompatible with the low anisotropy of the solid-liquid interfacial energy measured recently in aluminum alloys [5]. Second, using FIB reconstruction and observation, small zinc-rich particles have been found all along the twin plane of twinned $\mathrm{Al}-30 \mathrm{wt} . \% \mathrm{Zn}$ dendrites. In this case, the particles are located at the ledges of the partially coherent twin plane. This finding can be put into relation with the simulations which show an increase in solute-content along the liquid pool of the doublon and the formation of enriched liquid pockets at the root of the doublon. It is also probably linked with the small composition oscillations measured in a direction parallel to the twin plane near a twinned dendrite tip that was stopped by an equiaxed grain. Finally, a chemical analysis performed on a very thin Al-30 wt. \% Zn specimen extracted from a twinned dendrite trunk reveals a positive solute segregation near the twin plane, in agreement with the simulations and with the previous observations of Henry using a similar technique [2].

Although there is some evidence to indicate that the doublon is the stable growth morphology of twinned dendrites, a number of important questions still remain to be explained. Why do we observe the formation of zinc-rich particles along the twin plane of $\mathrm{Al}-\mathrm{Zn}$ specimens but no particles for Al-Cu alloys? Are these zinc particles formed during solidification or during solid-state transformations upon cooling, when crossing the miscibility gap or the two-phase region of the $\mathrm{Al}-\mathrm{Zn}$ phase diagram? What are the growth mechanisms of the ledges in the case of AlZn alloys?

\section{Acknowledgements}

The authors would like to thank Fabienne Bobard and the staff of the Interdisciplinary Center of Microscopy of the Ecole Polytechnique Fédérale de Lausanne (EPFL).

\section{References}

[1] Eady JA, Hogan LM. J Cryst Growth 1974;23:129-36.

[2] Henry S. PhD thesis, Ecole Polytechnique Fédérale de Lausanne; 1999.

[3] Wood HJ, Hunt JD, Evans PV. Acta Mater 1997;45(2):569-74.

[4] Salgado-Ordorica MA, Desbiolles J-L, Rappaz M. In: Crockcroft S, Maijer D, editors. Modelling of casting welding and advanced solidification processes, vol. XII. TMS; 2008. p. 545-52.

[5] Napolitano RE, Liu S. Phys Rev B 2004;70:214103.

[6] A Salgado-Ordorica M, Rappaz M. Acta Mater 2008;56:5708-18.

[7] Salgado-Ordorica M-A, Phillion A, Rappaz M, submitted for publication

[8] Salgado-Ordorica MA, Valloton J, Rappaz M. Scripta Mater 2009;61:367-70.

[9] Kurz W, Giovanola B, Trivedi R. Acta Metall 1986;34(5):823-30.

[10] Salgado-Ordorica MA, Desbiolles J-L, Rappaz M. Acta Mater 2011. doi:10.1016/i.actamat.2011.04.033.

[11] Salgado-OrdoricaMA. Ecole Polytechnique Fédérale de Lausanne; 2009.

[12] Straumal B, Kotgenkova O, Protasova S, Mazilkin A, Zieba P, Czeppe T, et al. Mater Sci Eng, A 2008;495:126-31.

[13] Straumal BB, Kogtenkova O, Zieba P. Acta Mater 2008;56:925-33.

[14] Cahn JW. J Chem Phys 1977;66:3667-72.

[15] Hamilton DR, Seidenticker RG. J Appl Phys 1960;31(1):1165-8.

[16] Rostamian A. PhD Thesis \#4336, Ecole Polytechnique Fédérale de Lausanne, Lausanne, Switzerland; 2009.

[17] Dey SR, Hazotte A, Bouzy E. Intermetallics 2009;17:1052-64.

[18] Watson MP, Hunt JD. Metall Mater Trans A 1977;8A:1793-800. 